\title{
El papel de las organizaciones de la sociedad civil en la reconfiguración de los programas sociales: análisis del caso argentino entre 2015 y 2019
}

\section{The role of civil society organizations in the reconfiguration of social programs: analysis of the Argentine case between 2015 and 2019}

Cynthia Ferrari Mango

FLACSO/CONICET-UNLaM (Argentina) ORCID: https://orcid.org/0000-0002-7416-6754 cferrari@flacso.org.ar

\begin{abstract}
NOTA BIOGRÁFICA
Licenciada en Ciencia Política (UNLaM). Magíster en Políticas Públicas (FLACSO). Doctora en Ciencias Sociales (UBA). Actualmente, es Becaria Posdoctoral de CONICET. Se desempeña como docente e investigadora en la UNLaM y en el Área de Estado y Políticas Públicas - FLACSO Argentina, donde también integra el Observatorio de Reforma Estructural y Políticas Públicas.
\end{abstract}

\section{RESUMEN}

En Argentina durante la gestión de la Alianza Cambiemos, la política social ha sido reconfigurada en sus concepciones potenciando o debilitando a actores territoriales vinculados a su implementación. En este sentido, nos proponemos analizar las estrategias que han llevado a cabo organizaciones para gestionar los programas del 2015 al 2019. Específicamente, nos concentramos en la posición que tomó el Movimiento Evita junto con la Confederación Argentina de Trabajadores de la Economía (CTEP) para gestionar el Argentina Trabaja - Programa Ingreso Social con Trabajo (AT-PRIST), que luego se reconvirtió en el Hacemos Futuro. Los mismos nos permiten observar particularidades de las políticas sociales, modalidades de gestión y la capacidad de agencia de los actores sociales. Sostenemos que a través de experiencias y prácticas, las organizaciones han capitalizado dichos programas en pos de las necesidades locales y han expandido su red territorial. La metodología que utilizamos es cualitativa y está basada en el trabajo de campo desde el año 2015 hasta el 2019, constituido por el análisis de normativas, revisión bibliográfica, pedidos de información al MDSN y entrevistas semiestructuradas.

\section{PALABRAS CLAVE}

Organizaciones sociales; Gestión; territorio; política social.

\begin{abstract}
In Argentina, during the management of the Alianza Cambiemos, social policy has been reconfigured in its conceptions, empowering or weakening territorial actors linked to its implementation. In this sense, we propose to analyze the strategies that organizations have carried out to manage the programs from 2015 to 2019. Specifically, we focus on the position taken by the Evita Movement together with the Argentine Confederation of Economic Workers to manage Argentina Work - Social Income with Work Program (ATPRIST), which later became the Make Future. They allow us to observe particularities of social policies, management modalities and the agency capacity of social actors. We maintain that through experiences and practices, organizations have capitalized on these programs in pursuit of local needs and have
\end{abstract}


GAPP. Nueva Época - N. 28, marzo 2022 - ISSN: 1989-8991 - DOI: https://doi.org/10.24965/gapp.i28.10877 - [Págs. 99-111]

El papel de las organizaciones de la sociedad civil en la reconfiguración de los programas sociales: análisis del caso argentino entre...

Cynthia Ferrari Mango

expanded their territorial network. The methodology we use is qualitative and is based on field work from 2015 to 2019, consisting of the analysis of regulations, bibliographic review, requests for information from the MDSN and semi-structured interviews.

\section{KEYWORDS}

Social Organizations; Management; territory; social policy.

\section{SUMARIO}

INTRODUCCIÓN. 1. RECONSTRUYENDO LA ESTRATEGIA METODOLÓGICA. 2. LAS ORGANIZACIONES COMO ACTORES EN LAS POLÍTICAS SOCIALES A PARTIR DE COOPERATIVAS. 3. LA OPORTUNIDAD COMPLEMENTÁNDOSE: EL «ARGENTINA TRABAJA» A PARTIR DE LAS ORGANIZACIONES. 4. LA RESISTENCIA CONFRONTÁNDOSE: EL «HACEMOS FUTURO» A PARTIR DE LAS ORGANIZACIONES. CONCLUSIONES. REFERENCIAS BIBLIOGRÁFICAS.

\section{INTRODUCCIÓN ${ }^{1}$}

Si bien a principios del siglo en varios países de América latina se desencadenó un «giro a la izquierda», en los últimos años se promovió un vuelco hacia la centro-derecha (Vommaro y Gené, 2017; Natanson, 2017). Específicamente, implicó el traspaso de gobiernos nacional-populares, neodesarrollistas y de centroizquierda, al ascenso de la derecha en países de América del Sur.

En Argentina durante los primeros años del siglo XXI, la reinclusión de población expulsada por el neoliberalismo se intentó a través de la generación de empleo revitalizando la industria, fomentando el consumo y promoviendo la autogestión y el cooperativismo. Esta última, fue una estrategia central dentro de la política social para la inclusión social y económica de población desocupada (Logiudice, 2018; Grassi, 2012; Dzembrowski y Maldovan Bonelli, 2010). En este marco, se combinaron estrategias bajo una matriz trabajo céntrica que generará empleo (Grassi, 2012) alejándose de los dispositivos del workfare e intentando superar la lógica de los programas de transferencias monetarias condicionadas (PTMC) a través de un discurso de economía social y promoción de actividades asociativas (Natalucci y Paschkes Ronis, 2011). Asimismo, devino en una política de Estado el fomento de experiencias autogestionadas por el movimiento de trabajadores desocupados (Hudson, 2016).

Específicamente, a partir del 2003 hasta el 2015 durante el gobierno del Frente para la Victoria (FPV) con un modelo de desarrollo productivo inclusivo (Garcia Delgado, 2013), desde un área del Ministerio de Desarrollo Social de la Nación (MDSN), revalorizaron el cooperativismo de trabajo y experiencias autogestionadas en torno a la centralidad del territorio (Perelmiter, 2012). En este marco, se creó el Argentina Trabaja - Programa Ingreso Social con Trabajo (AT-PRIST) destacándose por su masividad, magnitud presupuestaria y expansión territorial vinculada a municipios y organizaciones sociales ligadas a su gestión (Kasparian, 2019; Muñoz, 2019; Natalucci, 2018).

El objetivo del programa era generar inclusión social a través de la figura de la cooperativa de trabajo, de modo de aglutinar y fortalecer a personas que se encontraban excluidas del mercado laboral formal (Resolución 3182/2009 y Decreto N. 1067/09). Lo consideramos como una política pública en materia de inclusión social y generación de ingresos post-salariales que intervino en la distribución primaria del ingreso, a través de la conformación de cooperativas de trabajo (Kasparian, 2019; Hudson, 2016).

Tras la finalización del gobierno progresista y el inicio de una gestión conservadora con la Alianza Cambiemos promoviendo un modelo corporativo en el marco del neoliberalismo tardío (García Delgado y Gradin, 2017), a partir de la gestión del MDSN a cargo de Carolina Stanley, se reorganizan programas de economía social modificando su orientación (Ferrari Mango, 2021a; Muñoz, 2019; Hopp, 2018). Si bien la implementación del AT-PRIST continuó, atravesó modificaciones tales como la incorporación de nuevos entes ejecutores vinculados a organizaciones sociales (Resolución 592/2016). De este modo, se produce una oportunidad política (Tarrow, 1997; Tilly, 1978) para las organizaciones sociales que fortalecen la visión de economía popular. En efecto, se potencian experiencias reivindicativas a través de organizaciones sociales

\footnotetext{
1 Agradezco los editores y a los dos revisores anónimos sus comentarios sugerencias que han contribuido a la mejora del artículo.
} 
GAPP. Nueva Época - N. 28, marzo 2022 - ISSN: 1989-8991 - DOI: https://doi.org/10.24965/gapp.i28.10877 - [Págs. 99-111]

El papel de las organizaciones de la sociedad civil en la reconfiguración de los programas sociales: análisis del caso argentino entre...

Cynthia Ferrari Mango

que indicen en la agenda pública y adquieren una representación sindical desde la Confederación Argentina de Trabajadores de la Economía (CTEP) (Natalucci, 2018; Pacífico, 2018; Arcidiácono y Bermúdez, 2018; Maldovan et al., 2017).

A principios del 2018 la política social atraviesa un cambio de concepción que se visualiza tras la reconfiguración del AT-PRIST en el Hacemos Futuro (Res. N. ${ }^{\circ}$ 96/2018). El propósito del programa era empoderar a las personas promoviendo su autonomía económica a través de la terminalidad educativa, cursos y prácticas de formación integral que potencien sus posibilidades de inserción laboral (Res. 96/2018). La nueva propuesta se alejó de la concepción de la economía social que promovía cooperativas y produjo un reordenamiento en la organización del trabajo de las organizaciones sociales (Hintze, 2018; Ferrari Mango y Campana, 2018).

De este modo, a partir de la reconfiguración del programa y partiendo de la propia recursividad de la política social (Adelantado, Noguera, Rambla y Sáez, 1998), nos interrogamos en torno a ¿Qué particularidades adquirieron las estrategias que llevaron a cabo las organizaciones sociales en el territorio en función de la transformación del AT-PRIST en el Hacemos Futuro? En definitiva, el objetivo de este artículo es analizar desde la mirada de las organizaciones sociales los cambios en la implementación de la política de inclusión socio laboral. Específicamente, nos proponemos analizar las estrategias que han llevado a cabo las organizaciones sociales, haciendo hincapié en el Movimiento Evita junto con la CTEP, para gestionar el AT-PRIST y el Hacemos Futuro durante la gestión de la Alianza Cambiemos.

Por todo lo expuesto, el trabajo pretende brindar un aporte sobre los estudios de implementación de programas sociales en el territorio a través de la gestión de organizaciones sociales y la multiplicidad de factores que inciden. De esta forma, dejamos de considerar al diseño y a la implementación de una forma aislada para entenderlos como parte de reapropiaciones locales donde cobra relevancia la capacidad de agencia de los actores y las particularidades de su accionar en torno a complementariedad o bien confrontación. También, identificamos desde una mirada diacrónica el análisis de dos estrategias de implementación diferenciadas durante la misma gestión de gobierno que se distingue de la anterior alterando articulaciones y estrategias de los actores territoriales.

A partir de aquí el artículo se organiza en cinco apartados. En el primer apartado presentamos la estrategia metodológica de manera detallada. En el segundo nos enfocamos en delimitar el marco conceptual en torno a políticas sociales, actores, territorio y cooperativas. El tercer y cuarto apartado se dedican al desarrollo de los programas «Argentina Trabaja» y «Hacemos Futuro» presentando oportunidades y resistencias para las organizaciones sociales a través de los mismos. Por último, presentamos las conclusiones.

\section{RECONSTRUYENDO LA ESTRATEGIA METODOLÓGICA}

La metodología que utilizamos es cualitativa con técnicas basadas en el análisis de documentos, revisión bibliográfica y, principalmente, en el trabajo de campo. Este último los desarrollamos desde el 2016 hasta mediados del 2019 con énfasis en un distrito de la Provincia de Buenos Aires. Las técnicas de investigación cualitativas utilizadas se diferencian de acuerdo a dos niveles de análisis de la investigación, uno macro y otro micro que se complementaron y retroalimentan. En efecto, nos alejamos de visiones que los observen como compartimientos estancos, sino que dialogan entre sí.

A nivel macro institucional sistematizamos y analizamos normativas, documentos estales e información vinculada a los programas en la página web del AT-PRIST y del Hacemos Futuro (Res. 8132/2009, Res. 456/2016, Res. 592/2016, Res. 2055/2016, Res. 96/2018 y Res. 151/2018). A nivel micro sociopolítico, en una primera instancia llevamos a cabo la aproximación, identificación de los sujetos y de las redes sociales en el territorio. Para ello, combinamos las técnicas del informante clave con la técnica de bola de nieve (Guber, 1991). Luego, realizamos entrevistas semiestructuradas, las cuales tuvieron como índices temáticos las categorías e indicadores que operacionalizan los objetivos de la investigación (Guber, 2011; Bernard 1988; Taylor y Bogdan, 1987).

En una segunda instancia, para identificar a los sujetos a entrevistar realizamos un pedido de información al MDSN consultando cuáles eran los entes ejecutores desde el 2016 al 2018 en el recorte espacial seleccionado y con qué cantidad de titulares contaban. Esta información nos permitió equipararla con los resultados que veníamos recogiendo del trabajo de campo y terminar de definir en qué ente ejecutor nos íbamos a centrar parar analizar la reconfiguración a nivel territorial tras el cambio en la política social. El total de entrevistas que realizamos fue de once. Específicamente, los sujetos que entrevistamos fueron: 1.- tres 
GAPP. Nueva Época - N. 28, marzo 2022 - ISSN: 1989-8991 - DOI: https://doi.org/10.24965/gapp.i28.10877 - [Págs. 99-111]

El papel de las organizaciones de la sociedad civil en la reconfiguración de los programas sociales: análisis del caso argentino entre...

Cynthia Ferrari Mango

Cooperativistas/titulares en el marco de convenio entre Ministerio y Cooperativa de Trabajo Movimiento Evita La Matanza 1 Ltda. representada ante el MDSN por la Confederación de Trabajadores de la Economía Popular; 2.- tres representantes del ente ejecutor de la Cooperativa de Trabajo Movimiento Evita La Matanza 1 Ltda y de CTEP; 3.- dos funcionarios del MDSN de la gestión de la Alianza Cambiemos; 4.- tres funcionarios del Centro de Atención Local que funcionaba como burocracia territorializada el ministerio vinculado al ATPRIST y; tres funcionarios de la Administración Nacional de la Seguridad Social (ANSES) que se convirtió en la burocracia vinculada al Hacemos Futuro tras la desarticulación del CAL.

La mayoría de las entrevistas fueron grabadas y los textos obtenidos se analizaron de acuerdo a las técnicas de análisis de narrativas y de contenidos. Los datos también fueron registrados en notas de campo, y analizados por categorías e indicadores, a través de la discriminación y diferenciación de su contenido (Sautú, 2003; Scribano, 2000; Valles, 2000). También, realizamos observación participante (Guber, 2011) en el territorio en los espacios en los que se implementaba el programa.

\section{LAS ORGANIZACIONES COMO ACTORES EN LAS POLÍTICAS SOCIALES A PARTIR DE COOPERATIVAS}

La política social nos permite vislumbrar la interacción entre los actores sociales y el Estado en tanto instancia de articulación de relaciones sociales. El campo de las mismas está atravesado por luchas y enfrentamientos de diferentes fuerzas. Los actores se desenvuelven en un marco de institucionalidad en el cual las demandas sociales, que se transforman en políticas públicas, otorgan organicidad al aparato estatal y se materializan en instituciones, leyes, prácticas y procedimientos (Fleury, 1999).

A la política social la comprendemos como un instrumento para alterar los resultados negativos del mercado a través de una intervención que corrige o compensa la desigualdad social (Adelantado et al., 1998). En este sentido, genera entramados de actores colectivos que son claves para la explicación de su surgimiento y consolidación. Su influencia sobre los actores se observa contribuyendo a crear nuevos o destruirlos, incrementando o disminuyendo su capacidad de organización o su grado de institucionalización, movilizándolos o desmovilizándolos y distribuyendo directamente recursos económicos y de poder (Adelantado et al., 1998).

Uno de los actores en los que nos centramos son las organizaciones sociales que construyen instancias colectivas ejerciendo una representación tanto social como política que profundiza la ciudadanía y genera lazos comunitarios (De Piero, 2005). Partimos de identificar tres corrientes de organizaciones sociales a partir de diferenciar las modalidades de intervención, el grado de articulación con el Estado y los objetivos que persiguen tras la participación en las políticas públicas (De Piero, 2005). La corriente control-beneficencia demanda el control del Estado y asistencia. La corriente de protesta tiene una relación confrontativa y demandante con el sistema político dado que reclama inclusión y derechos sociales. La corriente de la economía social demanda auto resolución y desarrollo fortaleciendo alianzas con el Estado y el Mercado. El caso que utilizamos en este trabajo de la Cooperativa del Movimiento Evita que tiene representación en Confederación de Trabajadores de la Economía Popular (CTEP) lo ubicamos entre la segunda y tercer corriente.

Paralelamente, consideramos que el abordaje debe contener una dimensión relacional que resalte su vinculación con el Estado. En este sentido, las organizaciones sociales actúan como mediadoras gestionando la política social (Natalucci, 2018). La gestión de políticas sociales en el territorio adquiere una forma de politización produciendo una intercambios de bienes materiales e inmateriales (Auyero, 2001).

En efecto, identificamos a las organizaciones sociales como actores involucrados en procesos de gestión. Específicamente, observamos quiénes son aquellas que están vinculadas, cuáles son los objetivos que persiguen y cómo se articulan. Las mimas pueden participar en diferentes momentos de la política, aunque en este trabajo nos interesa particularmente la etapa de la implementación. Partimos del concepto de régimen de implementación local de Chiara y Di Virgilio (2006) que conjuga el modelo top down y el modelo bottom up (Aguilar Villanueva, 2003; Meny y Thoenig, 1992; Tamayo Sáez, 1997). Al mismo, lo entendemos como un conjunto de condiciones que repercuten en arreglos entre los actores y reconfiguran el diseño del programa en la implementación (Chiara y Di Virgilio, 2006).

En este trabajo, la organización social que identificamos en el momento de implementación a nivel territorial del AT-PRIST es la Cooperativa del Movimiento Evita que tiene representación en CTEP en calidad de ente ejecutora. Luego, en la reconfiguración del AT-PRIST al Hacemos Futuro, de ente ejecutor se transforma en Unidades de Capacitación (UCAPS). 
GAPP. Nueva Época - N. 28, marzo 2022 - ISSN: 1989-8991 - DOI: https://doi.org/10.24965/gapp.i28.10877 - [Págs. 99-111]

El papel de las organizaciones de la sociedad civil en la reconfiguración de los programas sociales: análisis del caso argentino entre...

Cynthia Ferrari Mango

Dicha cooperativa se enmarca en un discurso estatal de promoción de la economía social y solidaria para la creación de empleo y estímulo de participación colectiva. Asimismo, se materializa como espacio de inserción de movimientos sociales reivindicatorios de derechos y necesidades (Hintze, 2016; Kasparian, 2019). De este modo, oscila entre la tipología de cooperativa reivindicativa e inducida (Vuotto, 2011).

\section{LA OPORTUNIDAD COMPLEMENTÁNDOSE: EL «ARGENTINA TRABAJA» A PARTIR DE LAS ORGANIZACIONES}

Durante la gestión del Frente para la Victoria, en el AT-PRIST predominaba una orientación de economía social y solidaria, que fomenta el cooperativismo a través de la figura de cooperativa de trabajo e impulsaba la construcción colectiva, organizada y politizada de los actores locales. En la implementación del programa hasta el 2016 las organizaciones sociales no tenían convenio directamente con ministerio, sino que para la realización de la contraprestación necesitaban articular con el municipio. Específicamente, en lo que refería a la posibilidad de elegir en donde llevar a cabo sus funciones y recibir tanto herramientas como materiales de trabajo. Eva muestra el cambio: «Antes se le daba a los Municipios y ellos repartían y en el 2016 se armaron entes ejecutores. O sea se podía conveniar directamente con el Ministerio sin depender del Municipio» (Eva, Cooperativista Movimiento Evita, 08-03-2019).

En este marco, la Alianza Cambiemos decidió desplazar a los Municipios y articular directamente con organizaciones sociales con diferentes perfiles. Elena nos explica,

«A principios de 2016 se arma una mesa de diálogo con el compromiso del gobierno nacional de no darle de baja a ninguna persona, de poder seguir trabajando la línea del Argentina Trabaja y ahí nosotros quisimos ir por más. Nuestra propuesta fue que las organizaciones sociales podamos gestionar el programa en conjunto, definir las obras, bueno así se armaron distintos entes ejecutores con cooperativas que puedan realizar directamente el trabajo» (Elena, CTEP administrativa Movimiento Evita, 13-02-2019).

En este marco, la CTEP se ha posicionado como un actor de relevancia con quien negociar y es través del mismo que las organizaciones sociales, como el caso de Movimiento Evita obtuvieron la posibilidad de gestionar el AT-PRIST desde cooperativas conformadas en diferentes distritos. Las diferentes organizaciones, que conforman a la CTEP, son mencionadas por Alma como «patas del movimiento». Alma, lo ejemplifica del siguiente modo:

«Por ejemplo las patas que tenía era el Movimiento Evita era la Dignidad, Libertad o Pueblo Unido y el MTE. El Evita tenía una cantidad mucho mayor al resto, era por cantidad de gente, por espacio de la organización. Eran todos los mismos convenios. Lo que modificaba eran las zonas de incidencia y el nombre de quien firmaba digamos. Pero de nuevo después respondíamos frente a las negociaciones. Cuando hubo que negociar prórroga se negociaban todas juntas con la Mesa CTEP frente al Ministerio» (Alma, CTEP administrativa MTE, 15-12-2018).

En este sentido, construyeron un vínculo directo con el Ministerio a través de una mesa de gestión conformada por la CTEP lo cual les permitió tener «una línea directa de gestión, hay una mesa de gestión que se comunica o está en permanente comunicación con el MDSN» (Eva, Cooperativista Movimiento Evita, 08-032019). Una vez conformada la mesa CTEP, se realizaba una negociación con el MDSN. Elena cuenta que,

«la mesa de diálogo fue desde el principio con Stanley, que ya estaba designada en la cartera de desarrollo. Desde la CTEP asumo que habrá sido el gringo castro que es el secretario general de la CTEP, Emilio pérsico desde el Evita y Juan Grabois desde el MTE (movimiento de trabajadores excluidos) quien son como las organizaciones que estuvieron desde el principio en la CTEP» (Elena, CTEP administrativa Movimiento Evita, 13-02-2019).

En este sentido, con el cambio de gestión vieron una oportunidad y posibilidad de conveniar directamente con Ministerio. Para ello, tuvo una gran incidencia la CTEP desde donde se negocia con el MDSN los criterios cuantitativos y cualitativos vinculados al programa. Asimismo, tuvieron que institucionalizar la figura de la cooperativa. Elena ejemplifica,

«no en todos lados teníamos una cooperativa hecha y derecha. Nosotros tenemos una organización cooperativa pero que para el Estado no es una cooperativa porque no tiene libros, en- 
tonces fue regularizar todo eso. En principio ver que herramienta jurídica teníamos para poder llevar a adelante eso. Después agruparlo para que determinadas cooperativas coordinen el trabajo en varios lados» (Elena, CTEP administrativa Movimiento Evita, 13-02-2019).

Del relato de Eva, se vislumbra la herramienta cooperativa como instrumento para canalizar el trabajo. Asimismo, se desprende la diferencia de concepción de cooperativa que tenía el movimiento y la que pregonaba el programa.

Una vez firmado el convenio, se armó una estructura para llevarlo a cabo. En términos funcionales, se estableció una sede central administrativa de CTEP ubicada en constitución, Ciudad de Buenos Aires. Además, en el territorio se instauran diferentes entes ejecutores que eran «la cooperativa madre, el ente ejecutor, tenía todo un equipo que lo definía esa cooperativa distrital en conjunto con el resto de distritos si es que lo conformaban varios» (Elena, CTEP administrativo Movimiento Evita, 13-02-2019).

En lo que refiere a la sede administrativa Alma menciona que, «lo administrativo lo hacíamos desde la CTEP que es en el centro donde estábamos en constitución, Pedro Echague y Santiago del estero en primer piso» (Alma, CTEP administrativa MTE, 15-12-2018). Para la conformación de la sede administrativa tuvieron dificultades con el material de trabajo. Alma lo explica,

«Surge más desde el principio como pensado para Municipios e instituciones que estaban mucho más armadas de estructuras. Entonces cuando mostros fuimos a pedir comprar una computadora fue como todo un tema. O sea necesitábamos una computadora porque no teníamos donde sentar a nadie. Un escritorio o cosas muy básicas que el Ministerio nos decía está pensado para gente que ya tiene armada la estructura entonces nosotros no se la vamos a armar. Claro pero yo necesito imprimir cosas, algo muy básico» (Alma, CTEP administrativa MTE, 15-12-2018).

Alma nos brinda caracterizaciones del aspecto administrativo funcional más general,

«en CTEP coexiste organizaciones sociales está el Evita, está el MTE, está la dignidad, y demás y como CTEP en ese momento creo que había como 16 convenios y de esos 16 había uno de la dignidad, uno del MTE y todos los demás eran del Evita. Que tenían más trabajo acumulado y tenían la experiencia. Entonces ahí nosotros muy nuevos, arranco el convenio con un equipo más territorial armado pero sin nadie administrativo. Ahí me sumo yo a poner orden al caos que era eso fue cuando empecé a coordinar un poco ese equipo. En CTEP éramos no sé 5 ponele, y esas 5 personas teníamos 1.000 tareas. En la vida real éramos mucha más gente que 10 a tras de cada convenio» (Alma, CTEP administrativa MTE, 15-12-2018).

Además de establecer en el convenio las actividades a llevar a cabo y las personas destinadas a ello, realizaban otras tareas administrativas. Entre ellas, las vinculadas a recibir el material y el presupuesto. Para ello, tenían que presentar una rendición de los productos ya realizados que se había comprometido en el convenio.

En lo que refiere al territorio, plantea «teníamos los talleres donde funcionaban. Y después las organizaciones sociales tienen lugares en los territorios. Tiene no se los merenderos donde se ordenan y demás» (Alma, CTEP administrativa MTE, 15-12-2018). La estructura funcional a nivel territorial era la siguiente:

«había un equipo técnico digamos que en Argentina Trabaja eran digamos 10 personas estaba el titular del ente (el que comandaba), había después dos administrativos, una contable, uno técnico, cuatro coordinadores territoriales y dos promotores sociales. Maso menos era algo así» (Alma, CTEP administrativa MTE, 15-12-2018).

Las actividades estaban en enmarcadas en un plan de obra que seguía un esquema ya pre establecido por el MDSN. Alma aclara,

«cuando vos presentas el plan de obras tenías un modelo con talleres de herrería, carpintería, elaboración de dulces, bloquera, huerta y vivero. Cada taller tenía a 30 personas asignadas a eso. Vos presentas un proyecto de 300 personas y 15 talleres. Tenía ahí una correlación de cantidad de gente que presentabas y talleres. Vos todo lo aclaras de antes. Voy a hacer 10 paradas de colectivo en estas direcciones. Después podías hacer cambios con una nota de cambios» (Alma, CTEP administrativa MTE, 15-12-2018). 
GAPP. Nueva Época - N. 28, marzo 2022 - ISSN: 1989-8991 - DOI: https://doi.org/10.24965/gapp.i28.10877 - [Págs. 99-111]

El papel de las organizaciones de la sociedad civil en la reconfiguración de los programas sociales: análisis del caso argentino entre...

Cynthia Ferrari Mango

De este modo, existía un abanico de talleres en los cuáles podía participar los titulares del programa dentro de la cooperativa. Los mismos podían ser, herrería, carpintería, bloquera, elaboración de dulces, huerta y vivero. Tal como nos señalaba Alma, estaban como alternativas dentro de un modelo propuesto por MDSN en el cuál se seleccionaba el taller y se asignaban personas al mismo. Alma continúa explicándonos,

«Dentro de servicio comunitario tenías dos opciones que eran armados de paradas de colectivos, refacción de viviendas vulnerables, refacción de edificio comunes, desmalezamiento, cortar el paso, bueno un montón de cuestiones así menores que en los casos de municipalidades que tenían el Argentina Trabaja lo que hacían era poner su gente a cortar el paso en todo su Municipio. A nosotros nos interesaba llegar un poco más lejos. No presentamos proyecto de desmalezamiento sino que tenía más que ver con desmalezar para hacer una cancha por ejemplo. Mucho se fue a viviendas y edificios comunitario entonces así s e mejoraron el merendero, el comedor del barrio, la canchita del club, la casa de los vecinos, la vida cotidiana de los barrios» (Alma, CTEP administrativa MTE, 15-12-2018).

Del testimonio de Alma, observamos la diferencia que ella remarca con las actividades que llevaba a cabo el Municipio. También, resaltamos la relevancia que le adjudica a mejorar «la vida cotidiana de los barrios». De este modo, en el territorio los titulares realizaban tareas sociocomunitarias establecidas en el convenio. Para ello, se tomaba el presentismo a través de responsables sociales que eran los mismos titulares. Alma describe que,

«el presentimos era una lista que en cada uno de los territorios donde se daban los talleres vos tenes que decir toda esta gente vino. Era tarea de los dos responsables sociales de cada convenio. En la vida real lo que pasaba era que en cada taller pasaban lista y en los territorios era como que había uno que comandaba nose. Parada de colectivo. La pintura del playón no sé qué. Eran todos cooperativistas. Entonces si habías ido a trabajar de los 20 días laborales cobrabas el 100 si habías ido no sé 15 te pagaban 75 porciento» (Alma, CTEP administrativa MTE, 15-12-2018).

En lo que refiere a las tareas, que estaban previamente establecidas en los convenios, se resaltaba la vinculación con las necesidades del barrio. En este sentido, estaban vinculadas a problemáticas que ellos detectaban. Eva resalta la oportunidad de elegir dónde y que tareas realizar,

«se hicieron veredas en muchos lugares. Elegimos también donde poner una placita, donde poner recursos para hacer una plaza. La salita del barrio que nosotros queríamos ayudar o la es cuál del barrio que nosotros queríamos ayudar. Se generaron muchas actividades de ese tipo con el plan de actividades que nos dio independencia hacer esas cosas» (Eva, Cooperativista Movimiento Evita, 08-03-2019).

El ente ejecutor, particularmente las personas que lo materializan, se reapropiaron del programa y resignificaron su concepción de la política social. La concepción que intentan adjudicarle al programa es una visión emancipadora y estratégica. Observamos como intentan fortalecer al movimiento capitalizando para la organización, justamente en torno a la construcción de la organización. Elena recuerda,

«Ya en el 2011 decíamos que no nos Podemos quedar solo con los subsidios. Hay que armar una herramienta de los trabajadores. Todavía no teníamos del todo el concepto de economía popular pero si de trabajadores que no están reconocidos, que están fueras del sistema que se la arreglan como pueden, de la changa, de laburar en conjunto, de la fábrica recuperada, eso ya se empezó a gestar en ese momento» (Elena, CTEP administrativa Movimiento Evita, 13-02-2019).

Asimismo, como ente ejecutor adquiere particularidades de carácter combativo. En el caso de los movimientos se reconocen como compañeros desocupados vinculados a la organización que forjan lazos sociales para conseguir herramientas que permitan mejorar su calidad de vida. A modo de ejemplo, Elena anuncia,

«Nosotros vemos un reconfiguración muy diferente del capitalismo que es muy difícil volver al pleno empleo formal y lo que estamos planteando es una nueva forma de organizarse, una nueva forma de pensar el trabajo que es esto desde la organización popular con apoyo por supuesto del Estado» (Elena, CTEP administrativa Movimiento Evita, 13-02-2019). 
GAPP. Nueva Época - N. 28, marzo 2022 - ISSN: 1989-8991 - DOI: https://doi.org/10.24965/gapp.i28.10877 - [Págs. 99-111]

El papel de las organizaciones de la sociedad civil en la reconfiguración de los programas sociales: análisis del caso argentino entre..

Cynthia Ferrari Mango

De este modo enfatizan sobre el rol del trabajador de dicho espacio alejándose de visiones que lo consideren un asistido de un plan social. En definitiva, son vecinos y desocupados con objetivos y estrategias que potencian y reivindican a la economía popular.

\section{LA RESISTENCIA CONFRONTÁNDOSE: EL «HACEMOS FUTURO» A PARTIR DE LAS ORGANIZACIONES}

Tras la reconfiguración del AT-PRIST en el Hacemos Futuro, se elimina la figura de los entes ejecutores y algunos se reacomodan en Unidades de Capacitación (UCAPS). Las mismas son organizaciones gubernamentales o no gubernamentales que brindan capacitaciones a los titulares del Hacemos Futuro para que realicen su contraprestación. En base a un pedido de información al MDSN, tras preguntarles que son las UCAPS nos responden: «el objeto radica en brindar capacitaciones a los titulares del Programa Hacemos Futuro a fin de contribuir en su formación integral y futura inserción laboral, generando oportunidades de empleabilidad $»^{2}$. Este nuevo esquema lo explica una funcionaria del ministerio:

«Con el cambio de lo que es la resolución dejan de existir los entes ejecutores y empiezan a existir lo que son las UCPAS. Entonces se les dice: "bueno no se les va a bajar una determinada cantidad de dinero por cooperativista, pero tienen la posibilidad de brindar capacitación. Ustedes tienen que presentar un proyecto". Y no sé, después de determinada negociaciones se les iba a bajar dinero para que puedan capacitar» (Micaela, Funcionaria del CAL, 27-07-2018).

El relato de Micaela muestra la relevancia de las negociaciones para que el ente ejecutor se readapte en UCAPS y reciba financiamiento. Sostenemos que para la reconversión de ente ejecutor a UCAP, tuvo un rol fundamental las características del vínculo que habían tejido los actores con el MDSN en el período 20162017. A partir del 2018, se profundiza la direccionalidad del vínculo construido previamente.

En este sentido, se encargan de ofrecer capacitaciones previamente validadas por el MDSN. En lo que refiere a las particularidades del convenio, «los aportes a financiar por parte del Ministerio estarán afectados a insumos, capacitadores y seguro de responsabilidad civil, quedando el resto de los gastos inherentes al desarrollo de las actividades como aportes de las Unidades Capacitadoras» ${ }^{3}$.

Alma, nos detalla que se enteraron del cambio de modalidad repentinamente, aunque desde la organización de CTEP venían insistiendo en concretar reuniones con el MDSN dado que se estaba por vencer el convenio. Luego, de manera irónica explica: «nos juntamos con el Ministerio y nos contaron esta bella noticia de Hacemos Futuro y todo lo que eso implicaba» (Alma CTEP administrativa MTE, 15-12-2018).

Para transformarse en UCAPS volvieron a negociar través de CTEP los convenios, tal como lo habían hecho para convertirse en ente ejecutor. Las particularidades del convenio en el marco del Hacemos Futuro, establecían la condicionalidad de brindar capacitaciones. El inconveniente era que,

«Solamente se podía gastar plata en insumos, no nos permitía comprar maquinas. Y en algún arreglo de máquina, entonces si vos tenes un taller maso menos estabas en problemas y en los sueldos de los tallerista. Si nos permite ponele que se yo, comprar semillas, rastrillos, baldes, palas para trabajar la tierra» (Alma CTEP administrativa MTE, 15-12-2018).

De este modo, se desfinanciaba y se reducían los márgenes de acción. Concretamente, podían brindar cursos teóricos o bien talleres prácticos. Para ambos, utilizaron los soportes construidos anteriormente tanto desde su rol previo de ente ejecutor como de organización social que realizaba tareas sociocomunitarias. En lo que refiere a las formaciones teóricas, Alma nos expresa,

Ahí con las formaciones lo que hicimos fue algo piola porque CTEP tienen el ángulo formaciones, lo hacemos. Desde la militancia, ni siquiera por cumplir con un convenio entonces lo que veníamos haciendo se amoldo a eso y listo. Y salud paso a ser salud y adicciones. Acceso a la justicia paso a ser igualdad de género en la justicia. y cuestión ambiental paso a ser recicladores. Fue hablarlo con el Ministerio y que no hubiera discusión porque esto sabemos hacerlo (Alma CTEP administrativa MTE, 15-12-2018).

\footnotetext{
2 NO-2019-72641694-APN-CIIPES\#MSYDS 14 de Agosto de 2019- NO-2019-68074857-APN-DGAJMDS\#MSYDS, en relación a la solicitud de Acceso a la Información Pública.

3 NO-2019-72641694-APN-CIIPES\#MSYDS 14 de Agosto de 2019- NO-2019-68074857-APN-DGAJMDS\#MSYDS, en relación a la solicitud de Acceso a la Información Pública.
} 
GAPP. Nueva Época - N. 28, marzo 2022 - ISSN: 1989-8991 - DOI: https://doi.org/10.24965/gapp.i28.10877 - [Págs. 99-111]

El papel de las organizaciones de la sociedad civil en la reconfiguración de los programas sociales: análisis del caso argentino entre..

Cynthia Ferrari Mango

En este sentido, destaca como han institucionalizado experiencias que venían llevando a cabo y revaloriza el rol de la militancia social en la organización y en la formación. Algunos de los cursos que dictan son ${ }^{4}$ : promoción de la educación, promoción de la justicia, promoción de salud, trabajo, hábitat y ambiente. En lo que refiere a los talleres, Eva agrega:

«Nosotros tenemos una de huerta, una textil, una de una bloquera y panadería. Tres funcionan en el polo Palmar y una funciona en uno de los dentro culturales donde tenemos FINES porque ahí ya teníamos antes por el trabajo textil las maquinas entonces pudimos hacer que esa parte entre dentro del convenio. Ahí también tenemos secundario con oficio textil. Es medio como que para hacer secundario con oficios tenes que tener si o si las herramientas, las maquinas. Eso es en lo que se están capacitando todos los titulares que tienen que ver con Hacemos Futuro de nuestro espacio» (Eva, Cooperativista Movimiento Evita, 08-03-2019).

El testimonio de Eva, permite vislumbrar como continúan entablando lazos construidos con los titulares del programa que conforman también al movimiento. Específicamente, ella menciona «nuestro espacio». Paralelamente, gestionaron sedes en los barrios para dictar el FINES primaria, el FINES secundaria y secundaria con oficios.

La UCAP, recibía visitas de funcionarios del MDSN que realizan controles y están más presentes en regular la ejecución de las capacitaciones, en comparación con la etapa del programa anterior. Eva nos enuncia,

«Un técnico del Ministerio viene y observa mensualmente o en el momento que se les ocurre viene a ver como se llevan adelante esas tareas. Ellos hacen los informes. Esos informes llegan al Ministerio y de esa manera se comprueba si nosotros estamos cumpliendo o no con la tarea que tenemos asignada» (Eva, Cooperativista Movimiento Evita, 08-03-2019).

Además, la ANSES también funciona como un dispositivo de control dado que el certificado de realización del curso lo tenían que presentar en la agencia (Ferrari Mango, 2021b). Eva nos explica que,

«Los compañeros tienen que hacer capacitaciones de 4 meses. Después a través de ANSES se confirman si las hicieron o no. tienen que llevar el certificado de lo que están estudiando, que se llama FOTE o el CEFI No es que le dicen bueno, hagan las capacitaciones y los dejan librados al azar» (Eva, Cooperativista Movimiento Evita, 08-03-2019).

Para realizar los talleres en los cuales se entregan a los titulares el certificado, continuaron utilizando los espacios que usaban como ente ejecutor en el territorio que denominaban polos productivos. En este sentido, si bien hay una continuidad del espacio que utilizaban, se modifica la frecuencia de encuentro y el trabajo cotidiano.

En lo que refiere al cambio del programa, la lectura que hacen desde el movimiento es la despolitización y la desarticulación de las organizaciones. Alma relata,

«El Ministerio tiene un poco la lógica de las organizaciones sociales somos punteros todos. Y que ellos saben manejar a la gente. Entonces lo que querían hacer un poco era correrlos del medio y ellos encargasen de tener un vínculo directo con los. Entonces para corrernos del medio lo que buscaron era un vínculo directo» (Alma CTEP administrativa MTE, 15-12-2018).

De este modo persigue la búsqueda de desintermediarios. Otra representante de la organización, interpreta a la trasformación como la búsqueda de romper los lazos que las organizaciones habían construido con los titulares. Eva afirma que,

«Tenía que ver con desarmar o romper las organizaciones sociales que trabajaban o sea que sumaron gente a través de estos recursos. No todos los que tienen recursos de subsidios que genera el Estado participan en organizaciones sociales pero hay una gran cantidad que participan y eso también permite organizar la lucha que tiene que ver con los reclamos y los pedidos. La idea de modificar esto fue el desfinanciamiento y la desorganización de todo lo que podía ser oposición en la calle» (Eva, Cooperativista Movimiento Evita, 08-03-2019).

4 Fuente: En respuesta a la NO-2019-11902794-APN-DGAJMDS\#MSYDS, en relación al Pedido de Acceso a la Información Pública, en el marco de la Ley 27.275. 
GAPP. Nueva Época - N. 28, marzo 2022 - ISSN: 1989-8991 - DOI: https://doi.org/10.24965/gapp.i28.10877 - [Págs. 99-111]

El papel de las organizaciones de la sociedad civil en la reconfiguración de los programas sociales: análisis del caso argentino entre...

Cynthia Ferrari Mango

A pesar de la búsqueda de desorganizar y despolitizar, Eva nos resignifica el ideario de libertad para explicar cómo el titular elige seguir perteneciendo a la organización. Específicamente, en aquellos casos donde existía un relación social y un vínculo consolidado,

«Tu relación con el resto es si queres vas y sino queres no vas. Si queres te sumas y seguís participando de la lucha y sino no. No hay nadie que te va a obligar porque el recurso es entre Ministerio y vos. La organizaciones un intermediario que no te puede presionar de ninguna manera. También tiene sus contras porque te forma individual si vos en algún momento tenes algún problema para presentar los requerimientos que te dicen no va a ver nadie que pele por vos» (Eva, Cooperativista Movimiento Evita, 08-03-2019).

En la misma línea, Alma nos relata la percepción de una funcionaria del MDSN que se reincorpora al mismo con el cambio de gestión, «nos lo dijeron literalmente "a vos lo que te jode es que la gente pueda elegir que hacer"» (Alma CTEP administrativa MTE, 15-12-2018). En definitiva, se desprende una lectura negativa en tono al clientelismo desde los funcionarios del MDSN, al intentar con la transformación del programa eliminar a los intermediarios. Sin embargo, los entes ejecutores se han transformado en UCAP, aunque tienen menos margen de acción para poder llevar a cabo tareas sociales en los barrios y menor presupuesto. Por ello, hablamos de un recorte y vaciamiento de soportes del programa. No obstante, a través del Salario Social Complementario (SSC) gestionado desde la CTEP, continúan teniendo presencia en tareas sociocomunitarias en el territorio. Eva explica,

«Todos los que trabajan y reciben este beneficio se organizan en unidad productivas en las que realizan diferentes tareas. Por ejemplo, van a pintar escuelas, se ocupan de la placita del barrio, colaboran en los merenderos, en los roperitos, organizan actividades sociales cuando hay inundaciones, participan en todas esas cosas. Las personas que están dentro del Hacemos futuro no se les pide que hagan tareas de este tipo, las hacina antes cuan do el programa era Argentina Trabaja» (Eva, Cooperativista Movimiento Evita, 08-03-2019).

El relato de Eva, permite vislumbrar como se reacomodaron a pesar de las estrategias de desintermediación promovidas a través del Hacemos Futuro. Para readaptarse en UCAP, sostenemos que incidió el vínculo contraído en la etapa anterior cuando el programa comienza a implementarse por organizaciones además de por municipios.

\section{CONCLUSIONES}

La relación entre las organizaciones sociales y la gestión de programas sociales ha adquirido un carácter de recursividad tras las reconfiguraciones en la concepción de la política social así como también por la capacidad de agencia de los actores colectivos. Desde un enfoque amplio de la economía social, la figura de la cooperativa de trabajo es la que materializa la implementación local del programa. Específicamente, desde la mirada de las organizaciones sociales los cambios formulados en la implementación del Argentina Trabaja y su reconversión en el Hacemos Futuro implicaron en el inicio del gobierno de la Alianza Cambiemos una oportunidad, que luego se reconvirtió en una resistencia confrontada.

El artículo nos permite comprender la direccionalidad de la política social durante la gestión de un gobierno conservador y la capacidad de agencia de las organizaciones frente al mismo. Específicamente, como el Movimiento Evita junto con la CTEP logró adaptarse para dar materialidad a los programas sociales en función de las necesidades de los territorios. Las organizaciones sociales enmarcándose en la CTEP han logrado capitalizar los programas Argentina Trabaja y Hacemos Futuro en favor de las necesidades de los barrios que representan. Ambos programas han permitido extender una trama territorial que se concentró en responder y atender a poblaciones excluidas. Paralelamente, fortalecieron y consolidaron al movimiento mostrando la relevancia de las redes que construyen las organizaciones sociales en virtud de mejorar la calidad de vida en los barrios.

La figura institucional bajo la cual se organiza el trabajo fueron diversas cooperativas reglamentadas que se caracterizaban por promover un tipo de gestor combativo, cooperativo y estratégico con una visión emancipadora y un titular como compañero desocupado. Con la transformación del Argentina Trabaja en el Hacemos Futuro si bien se redujeron los soportes institucionales colectivos, la organización logró readaptarse. Específicamente, el instrumento de ente ejecutor se reconfiguró en UCAPS. Para dicha transformación 
GAPP. Nueva Época - N. ${ }^{\circ}$ 28, marzo 2022 - ISSN: 1989-8991 - DOI: https://doi.org/10.24965/gapp.i28.10877 - [Págs. 99-111]

El papel de las organizaciones de la sociedad civil en la reconfiguración de los programas sociales: análisis del caso argentino entre...

Cynthia Ferrari Mango

fue fundamental el vínculo que la organización había construido tanto con funcionarios del MDSN como con los titulares del programa. El primero permitió una continuidad en el marco formal, aunque desde la figura de UCAPS. El segundo mostró el lazo consolidado entre la organización y el titular. En dicho proceso se institucionalizaron prácticas de capacitación que las organizaciones ya venían desarrollando con el objetivo de concientizar y debatir entre los titulares en torno a diversas temáticas. En este sentido, lo relevante es que muchas UCAPS son el resultado de la mutación de los entes ejecutores.

Con la finalización del gobierno de la Alianza Cambiemos y la asunción del Frente de Todos se reconfigura el Hacemos Futuro en el Potenciar Trabajo. Dicha modificación nos genera nuevos interrogantes tales como ¿qué estrategias llevarán a cabo los actores territoriales sean organizaciones sociales o bien los municipios? ¿Qué tipos de proyectos se impulsarán? ¿Cómo se implantarán? ¿Qué continuidades y discontinuidades se observaran?

\section{REFERENCIAS BIBLIOGRÁFICAS}

Adelantado, J., Noguera, J. A., Rambla, X. y Sáez, L. (1998). Las relaciones entre estructura y política sociales: una propuesta teórica. Revista Mexicana de Sociología, 60(3), 123-156.

Aguilar Villanueva, L. (2003). Estudio Introductorio. En L. Aguilar Villanueva (ed.), La hechura de las Políticas. MAPorrúa.

Arcidiácono, P. y Bermúdez, Á. (2015, 5-7 de agosto). La expansión del cooperativismo de trabajo bajo programas. Una mirada sobre el Programa Ingreso Social con Trabajo-Argentina Trabaja [Ponencia]. En XII Congreso Nacional de estudios del trabajo. El trabajo en su laberinto. Viejos y nuevos desafíos. Asociación Argentina de especialistas en estudios del trabajo. https://aset.org.ar/congresos-anteriores/12/ponencias/11_Arcidiacono.pdf

Arcidiácono, P. y Bermúdez, Á. (2018). ¿Cooperativismo como oportunidad perdida? Problemas estructurales y coyunturales del cooperativismo bajo programas. Revista Ciudadanías. Revista de Políticas Sociales Urbanas, 2 , 83-111. http://revistas.untref.edu.ar/index.php/ciudadanias/article/view/534

Auyero, J. (2001). La política de los pobres. Ediciones Manantial.

Bernard, R. (1988). Research methods in Cultural Anthropology. Sage.

Chiara, M. y Di Virgilio, M. (2006). La política social en la crisis de la convertibilidad (1997-2001): mirando la gestión desde las coordenadas municipales en el Gran Buenos Aires. En Andrenacci, L. (comp.), Problemas de la política social en la Argentina contemporánea (pp. 125 a 156). Prometeo.

De Piero, S. (2005). Organizaciones de la sociedad civil. Editorial Paidós.

Dzembrowski, N. (2018). Fábricas recuperadas: Ios sentidos de la organización cotidiana del trabajo. Trabajo y sociedad: Indagaciones sobre el empleo, la cultura y las prácticas políticas en sociedades segmentadas, 30, 141-154. https://www.unse.edu.ar/trabajoysociedad/30\%20DSZEMBROWSKI\%20fabricas\%20recuperadas $\% 20$ los\%20sentidos.pdf

Dzembrowski, N. y Maldovan Bonelli, J. (2010). La asociatividad para el trabajo como productora de lazos sociales: un análisis de sus dimensiones a partir de dos tipos asociativos en la Argentina actual. En C. Cross y M. Berger (comps.), La producción del trabajo asociativo. Condiciones, experiencias y prácticas en la economía social (pp. 129-150). CICCUS.

Ferrari Mango, C. (2021a). Aportes para una periodización: del Argentina Trabaja al Hacemos Futuro. Estudios Sociales Contemporáneos, 24, 163-187. https://doi.org/10.48162/rev.48.007

Ferrari Mango, C. (2021b). Burocracia social: reconfiguración macroinstitucional y micro sociopolítica entre la ANSES y el programa Hacemos Futuro. Población \& Sociedad, 28, 86-106. http://dx.doi.org/10.19137/pys-2021-280105

Ferrari Mango, C. (2019a). Territorio, política social e implementación. Una mirada desde los espacios locales de gestión del Argentina Trabaja - Programa Ingreso Social con Trabajo en el distrito de La Matanza. Ciudadanías. Revista de Políticas Sociales Urbanas, 4, 203-232. http://revistas.untref.edu.ar/index.php/ciudadanias/article/ view/509

Ferrari Mango, C. (2019b). Política Social Desde Cooperativas Reguladas: Argentina Trabaja - Programa Ingreso Social Con Trabajo. Revista de ciencias sociales de la Universidad de Costa Rica. 165, 121-133. https://doi. org/10.15517/RCS.V0I165.40068

Ferrari Mango, C. (2019c). La política pública y sus elementos constitutivos: una mirada desde el Programa Ingreso Social con Trabajo - Argentina. Revista Trabajo y Sociedad, 33, 349-369, https://www.unse.edu.ar/ trabajoysociedad/33\%20FERRARI\%20MANGO\%20politicas\%20publicas\%20Argentina\%20Trabaja.pdf

Ferrari Mango, C. y Campana, J. (2018, julio). Del «Argentina Trabaja - Programa Ingreso Social con Trabajo» y el "Ellas Hacen» al "Hacemos Futuro». ¿Integralidad o desintegración de la función social del Estado? [Informe n. ${ }^{\circ} 11$ del Observatorio sobre Políticas Públicas y Reforma Estructural]. https://politicaspublicas.flacso.org. ar/2018/06/03/informe11/

Fleury, S. (1999). Políticas sociales y ciudadanía. Banco Interamericano de Desarrollo - Instituto Interamericano para el Desarrollo Social (INDES). 
GAPP. Nueva Época - N. 28, marzo 2022 - ISSN: 1989-8991 - DOI: https://doi.org/10.24965/gapp.i28.10877 - [Págs. 99-111]

El papel de las organizaciones de la sociedad civil en la reconfiguración de los programas sociales: análisis del caso argentino entre..

Cynthia Ferrari Mango

García Delgado, D. (2013). Editorial Estado y Políticas Públicas: hacia un nuevo paradigma. Revista Estado y Políticas Públicas, 1, 14-18.

García Delgado, D. y Gradin, A. (comps.)(2017). El neoliberalismo tardío. Teoría y praxis [Documento de Trabajo, n. ${ }^{\circ}$ 5]. FLACSO Argentina. https://www.flacso.org.ar/publicaciones/el-neoliberalismo-tardio-teoria-y-praxis/

Gradin, A. (2013, 6-8 de noviembre). Estado, dispositivos de gestión y territorio: la participación de los movimientos de desocupados en los programas de asistencia al desempleo. En VII Jornadas de Jóvenes Investigadores Instituto de Investigaciones Gino Germani.

Gradin, A. (2018). Estado, territorio y participación política. Editorial Teseo. https://www.flacso.org.ar/wp-content/ uploads/2018/11/Estado-territorio-y-participaci\%C3\%B3n-pol\%C3\%ADtica-GRADIN-Agustina-2018.pdf

Grassi, E. (2012). Política socio laboral en la argentina contemporánea. Alcances, novedades y salvedades. Revista Ciencias Sociales de la Universidad de Costa Rica, 135-136, 185-198. http://doi.org/10.15517/RCS.V0I135136.3676

Guber, R. (2011) La etnografía. Siglo XXI Editores.

Hintze, S. (2016). Potencialidades y riesgos de las cooperativas de trabajo en Argentina. Revista Temas, 87-88, $112-118$

Hintze, S. (2018). Políticas, asociatividad y autogestión en la Argentina post 2015. Otra Economía, 11(20), 136-155. https://www.revistaotraeconomia.org/index.php/otraeconomia/article/view/14737

Hopp, M. (2018). De la promoción del trabajo cooperativo al Salario Social Complementario. Transformaciones en la transferencia de ingresos por trabajo en la Argentina. Ciudadanías. Revista de Políticas Sociales Urbanas, 2, 113142. http://revistas.untref.edu.ar/index.php/ciudadanias/article/view/535

Hudson, J. (2016). Políticas públicas y empresas recuperadas por sus obreros en Argentina. Un análisis del Programa de Trabajo Autogestionado 2004-2012. Apuntes Revista de Ciencias Sociales, 79, 157-184. https://doi. org/10.21678/apuntes.79.869

Hudson, J. (2017). Gobiernos progresistas y autogestión en la Argentina 2003-2015: cooperativas no-estatales, sintéticas y anfibias. Revista Latinoamericana de Estudios del Trabajo, 21(34), 91-122. https://ri.conicet. gov.ar/bitstream/handle/11336/77225/CONICET_Digital_Nro.9ce025b1-0dc6-44b1-8f9a-7b5cf65b8acf_A. pdf?sequence $=2$

Kasparian, D. (2019). Promoción estatal del cooperativismo de trabajo y formas socioproductivas emergentes. RevIISE, 13(13), 211-225. http://www.ojs.unsj.edu.ar/index.php/reviise/article/view/313

Logiudice, A. (2017). La asistencia social actual: entre la aseguración y la activación de los trabajadores. En P. Arcidiácono y C. Zibecchi (coords.), La trama de las políticas sociales. Estado, saberes y territorio (pp. 83-110). Editorial Biblos.

Logiudice, A. (2018). Pasado y presente de la asistencia: claves para una relectura del neoliberalismo. Revista de la Carrera de Sociología, 8(8), 90-124. https://publicaciones.sociales.uba.ar/index.php/entramadosyperspectivas/ article/view/2967

Maldovan Bonelli, J., Fernández Mouján, L., Ynoub, E. y Moler, E. (2017). Los descamisados del siglo XXI: de la emergencia del sujeto trabajador de la economía popular a la organización gremial de la Ctep (2011-2017). Cartografías del Sur. Revista De Ciencias, Artes y Tecnología, (6), 41-64. https://cartografiasdelsur.undav.edu.ar/ index.php/CdS/article/view/87

Maneiro, M. (2018). La lucha de las organizaciones de trabajadores desocupados en los últimos años del kirchnerismo. Análisis del proceso de protesta ligado al Programa «Argentina Trabaja». Sociohistórica, 42, e058. https://doi. org/10.24215/18521606e058

Meny, Y. y Thoenig, J. (1992). Políticas públicas y teoría del Estado. En Y. Meny y J. Thoenig (comp.), Las políticas públicas (pp. 19-4). Ariel.

Muñoz, A. (2019). Voluntades populares, voluntades laborales. El caso de la Confederación de los Trabajadores de la Economía Popular. Trabajo y Sociedad, 32, 479-510. https://www.unse.edu.ar/trabajoysociedad/32 \%20 MUNOZ\%20MARIA\%20CTEP.pdf

Natalucci, A. (2012). Políticas sociales y disputas territoriales. El caso del programa «Argentina Trabaja». Perspectivas de Políticas Públicas, 2(3), 126-151. https://doi.org/10.18294/rppp.2012.614

Natalucci, A. (2018). El neoliberalismo en acto: políticas sociales y experiencias organizativas en Argentina (20092016). Polis (Santiago), 17(49), 103-125. https://polis.ulagos.cl/index.php/polis/article/view/1329

Natalucci, A. y Paschkes Ronis, M. (2011). Avatares en la implementación de políticas sociales. Concepciones y prácticas de las organizaciones sociopolíticas que participan en el programa Argentina Trabaja (2009-2010) [Trabajo seleccionado]. En IV Encuentro Internacional de Trabajo Social de la Universidad de Buenos Aires: Políticas Públicas y Trabajo Social, aportes para la reconstrucción de lo público (pp. 1-24). Universidad de Buenos Aires.

Natanson, J. (2017). La «ola amarilla» en Argentina: Reconfiguraciones tras el triunfo de Cambiemos. Nueva Sociedad, 272, 4-12. https://nuso.org/articulo/la-ola-amarilla-en-argentina/

Pacífico, F. (2018). Generarse el trabajo, construir para el barrio. Reflexiones en torno a «lo productivo» a partir de una experiencia de la economía popular en el marco de un programa social. En N. Goren y P. Isacovich (comps.), El trabajo en el Conurbano Bonaerense (pp. 87-115). Edunpaz. 
Perelmiter, L. (2012). Fronteras inestables y eficaces. El ingreso de organizaciones de desocupados a la burocracia asistencial del Estado. Argentina (2003-2008). Estudios Sociológicos de El Colegio De México, 30(89), $431-458$. https://doi.org/10.24201/es.2012v30n89.148

Rebón, J. y Kasparian, D. (2015). La valoración social de las cooperativas en el área metropolitana de Buenos Aires. Una aproximación a partir de la investigación por encuesta Cayapa. Revista Venezolana de Economía Social, 15(29), 11-37. http://www.saber.ula.ve/handle/123456789/41625

Sautú, R. (2003). Todo es teoría. Objetivos y métodos de investigación. Ed. Lumiere.

Scarfó, G., Hopp, M. y Highton, C. (2009). Reflexiones en torno al concepto de inempleabilidad: consideraciones para Pensar la política social. Tandil, 2(2), 8-20.

Scribano, A. (2000). Reflexiones epistemológicas sobre la investigación cualitativa en ciencias sociales. Cinta de Moebio. Revista de Epistemología de Ciencias Sociales, 8, 128-136. http://www.moebio.uchile.cl/08/scribano.html

Tamayo Saez, M. (1997). El Análisis de las Políticas Públicas. En R. Bañón y E. Carrillo (comps.), La Nueva Administración Pública. Alianza Universidad Textos.

Tarrow, S. (1997). El poder en movimiento. Los movimientos sociales, la acción colectiva y la política. Alianza.

Taylor, S. J. y Bogdan, R. (1987). Introducción a los métodos cualitativos. Paidós.

Tilly, C. (1978). From Mobilition to Revolution. McGraw Hill.

Valles, M. S. (2000). Técnicas cualitativas de investigación social. Síntesis.

Vommaro, G. y Gené, M. (2017). Argentina: el año de Cambiemos. Revista de ciencia política (Santiago), 37(2), 231254. http://ojs.uc.cl/index.php/rcp/article/view/5114

Vuotto, M. (2011). El cooperativismo de trabajo en la Argentina: contribuciones para el diálogo social [Documento de Trabajo, 217]. OIT. http://www.ilo.org/americas/publicaciones/WCMS_179395/lang--es/index.htm 